\title{
LONG-TERM CARDIOVASCULAR HEALTH IN ADULT CANCER SURVIVORS
}

W.R. Naaktgeboren $\mathrm{BSc}^{1}$, M. Linschoten $\mathrm{BSc}^{1}$, A. de Graeff MD $\mathrm{PhD}^{2}$, A. v Rhenen MD $\mathrm{PhD}^{3}$, M.J. Cramer MD PhD ${ }^{1}$, F.W. Asselbergs MD PhD ${ }^{1,4,5,6}$, A.H.E.M. Maas MD $\mathrm{PhD}^{7}$, A.J. Teske MD $\mathrm{PhD}^{1 *}$

1) Department of Cardiology, Division of Heart and Lungs, University Medical Centre Utrecht, Utrecht, the Netherlands

2) Department of Medical Oncology, University Medical Centre Utrecht, Utrecht, The Netherlands

3) Department of Medical Haematology, University Medical Centre Utrecht, Utrecht, The Netherlands

4) Farr Institute of Health Informatics Research and Institute of Health Informatics, University College London, London, United Kingdom

5) Durrer Center for Cardiovascular Research, Netherlands Heart Institute, Utrecht, the Netherlands;

6) Institute of Cardiovascular Science, Faculty of Population Health Sciences, University College London, London, United Kingdom;

7) Department of Cardiology, Radboud University Medical Centre, Nijmegen, The Netherlands

\section{Corresponding author}

Arco J. Teske, M.D. Ph.D.

Department of Cardiology, Division of Heart \& Lungs

University Medical Centre Utrecht

Room number F02.318, P.O. Box 85500

3508 GA Utrecht, the Netherlands

Phone: +31887574420

Email: a.j.teske@gmail.com 
$\underline{\text { List of abbreviations }}$

\begin{tabular}{|l|l|}
\hline AIC & Anthracycline-induced cardiotoxicity \\
\hline CAD & Coronary artery disease \\
\hline CI & Confidence interval \\
\hline CV & Cardiovascular \\
\hline CVD & Cardiovascular disease \\
\hline CHF & Congestive heart failure \\
\hline HER-2 & Human epidermal growth factor receptor-2 \\
\hline HR & Hazard ratio \\
\hline Gy & Gray \\
\hline LV & Left ventricular / Left ventricle \\
\hline LVEF & Left ventricular ejection fraction \\
\hline OR & Odds ratio \\
\hline RCT & Randomised controlled trial \\
\hline RT & Radiotherapy \\
\hline VEGF & Vascular endothelial growth factor \\
\hline VHD & Valvular heart disease \\
\hline
\end{tabular}

\section{Abstract}

The population of cancer survivors has tremendously increased over the past decades as a result of aging of the population and improvements in early cancer detection and treatment. Ongoing successes in cancer treatment are expected to result in a further increase of long-term survivors. However, cancer treatment can have detrimental cardiovascular side effects that impact morbidity and mortality, reducing quality of life in cancer survivors. The spectrum of radio- and chemotherapy-induced cardiovascular disease is broad, varying from subclinical valvular dysfunction to overt congestive heart failure and may not be apparent for more than twenty years after the initial cancer treatment. Awareness of these long-term side effects is of crucial value for the management of these patients, in order to reduce the impact of cardiovascular morbidity and mortality. This review provides a comprehensive overview of the long-term cardiovascular complications of cancer treatments (radiotherapy and chemotherapy) in adult cancer survivors.

Keywords: cardiotoxicity, cancer survivor, radiotherapy, chemotherapy, cardiovascular disease, cardio-oncology 


\section{Introduction}

Over the past decades, improvements in early cancer detection and treatment have significantly improved survival rates of many malignancies[1]. Furthermore, the incidence of cancer patients has increased (and will increase further) due to aging of the population[2]. As a result of these two trends, the population of cancer survivors is growing rapidly in the Western world. In 2012, nearly fourteen million cancer survivors were alive in the United States and it has been postulated that this will increase to eighteen million by 2022[2]. A similar trend is observed in Europe[3].

Within the population of cancer survivors, the awareness for health problems that can occur after cancer survival is increasing. Besides the risk of recurrent or secondary malignancies, functional disabilities and psychosocial distress, cancer survivors are prone to develop cardiovascular disease (CVD)[4,5]. Figure 1 provides an example on the characteristic survival pattern observed in survivors of Hodgkin's disease.[6] Compared to people without a cancer history, CVD risk is $30 \%$ higher in adult cancer survivors[7]. In addition, CVD is the most common cause of non-cancer death among cancer survivors and significantly reduces the eight-year overall survival from $81 \%$ to $60 \%$ compared to cancer survivors without CVD [8,9].

Traditionally, cancer and CVD were considered two different entities. However, there is growing evidence that there might be a common biological pathway, as cancer and CVD share common risk factors such as ageing, smoking and obesity[10]. Patients with these preexisting risk factors at baseline are also more likely to develop CVD during and after anticancer treatment[11]. Finally, preliminary evidence suggests that subclinical myocardial damage can occur prior to anticancer treatment, implicating an effect on cardiac function by the malignant process itself[12].

The spectrum of anticancer treatment-induced CVD is wide, varying from valvular heart disease (VHD) and constrictive pericarditis to ischaemic heart disease and overt congestive heart failure (CHF)[13]. The first clinical manifestations may appear more than twenty years after exposure[4]. Awareness of these long-term side effects is therefore of importance in the follow-up and management of these patients. This review provides a comprehensive overview of the long-term cardiovascular (CV) complications of anticancer treatments (radiotherapy, chemotherapy and targeted therapy) in adult cancer survivors. Acute CV complications of anticancer treatments in adults[14], as well as long-term side effects in childhood cancer survivors will be discussed elsewhere in this issue and are out of the scope of this review. 
2. Long-term radiotherapy-induced cardiovascular disease

Radiotherapy (RT) is an integral component of anticancer treatment in nearly $50 \%$ of cancer patients[15]. Exposure of the heart to RT has been associated with severe long-term CV complications in cancer survivors. In the 1960's, it was first recognised that thoracic RT might induce severe CV sequelae[16], which led to drastic refinements in RT techniques over the past decades. As a result, acute complications (i.e. acute pericarditis) have become rare and most RT-induced complications manifest ten to twenty years after exposure[17]. It is expected that the long term complications will be reduced by the implementation of more heart-sparing techniques.

Histopathological, the hallmark of RT-induced damage is fibrosis[18]. The underlying pathophysiological mechanism inducing fibrosis formation is not fully elucidated. Current theories are based on results from animal studies and suggest a complex combination of micro- and macrovascular pathology[15,19]. RT induces microvascular injury which eventually lead to loss of endothelial cells and endothelial dysfunction. Macrovascular damage enhances the acceleration of atherosclerosis, leading to narrowing of the lumen of coronary arteries and further deterioration of the endothelial function and ischaemia [20].

Even though RT can affect virtually every structural component of the CV system, the proximal aorta, coronary arteries, valves, pericardium, and conduction system are predominantly affected[17] (Figure 2). Small studies have also suggested regional functional myocardial dysfunction due to direct exposure to RT[21]. Risk factors for RT-induced CV complications are younger age at time of RT exposure, dose>30 Gray (Gy) or a fractional dose of $>2 \mathrm{~Gy}$, the use of concomitant cardiotoxic systemic chemotherapy (i.e. anthracyclines) and the presence of CV risk factors or pre-existing CVD at baseline [13]. As a result, RTinduced sequelae are predominantly seen in survivors of Hodgkin's disease and, to a lesser degree, survivors of breast cancer. Additionally, RT-induced complications are increasingly reported in survivors of oesophageal and lung cancer, as the survival rates of these malignancies are increasing[22,23].

In a large meta-analysis, pooling results of more than 45,000 survivors of Hodgkin's disease, breast cancer, lung cancer and metastatic testicular cancer, the risk of long-term heart failure was 1.8-fold increased for patients treated with mediastinal RT (95\% confidence interval (CI) 1.1-3.1)[24]. In survivors of Hodgkin's disease, the 40-year cumulative incidence for any CVD was 25\% (95\% CI 17\%-33\%) for patients not treated with mediastinal RT or anthracyclines, which more than doubled to 55\% (95\% CI 41\%-52\%) for patients treated with mediastinal RT alone [25]. 


\subsection{Coronary artery disease}

The association between RT and ischaemic heart disease was controversial for many years and first fully recognised after the publication of large epidemiologic studies in the 1990's on survivors of Hodgkin's disease and breast cancer[26,27]. In a retrospective Dutch cohort study of 2,524 survivors of Hodgkin's disease who were treated with RT between 1965-1995, a 40-year cumulative incidence for coronary artery disease (CAD) as first cardiac diagnosis after treatment of $23 \%$ (95\% CI 21\%-25\%) was reported[25]. Within this cohort, the majority of patients developed more than one cardiac event, of which CHF was the most prevalent secondary diagnosis [25]. Compared to the general population, the risk for angina pectoris and fatal myocardial infarction was 4.1 and 3.6-fold increased for survivors of Hodgkin's disease [4]. With respect to survivors of breast cancer, a large population-based case-control study in 2,168 women who underwent RT between 1958 and 2001, reported that women receiving left-sided RT had significantly higher rates of ischaemic heart disease compared to those receiving right-sided RT (mean dose to the heart for left- versus right-sided of $6.6 \mathrm{~Gy}$ and $2.9 \mathrm{~Gy}$ )[28]. The risk of major cardiac events increased linearly with $7.4 \%$ per Gy inflicted to the heart, emphasizing the need for heart-sparing techniques[28].

Although the mechanism of atherosclerotic plaque formation, -rupture and occlusion is similar as in non-radiated patients, RT-induced CAD seems to be slightly different from conventional CAD. Firstly, post-mortem examination of irradiated patients revealed that the plaques were more fibrous and contained less lipid components[18]. Secondly, plaques in irradiated patients are more often located in the ostium of arteries and tend be smoother, longer and more concentric than conventional atherosclerotic plaques[29]. Finally, the left descending coronary artery is most often affected[30] (Figure 3). As a result, percutaneous intervention in RT-induced CAD is more challenging. Importantly, irradiated cancer survivors can remain asymptomatic for a relatively long period of time, even in presence of severe CAD and sudden cardiac death may be the first clinical manifestation[31].

Official guidelines on screening and follow-up of cancer survivors at risk for CAD are lacking. However, according to preliminary guidelines/expert consensus, it is advised to start screening for CAD in patients who received thoracic RT five to ten years after exposure and repeat imaging every five year[20]. Imaging modality of choice is depended local preferences and availability, although cardiac CT might be preferably in a pre-operative setting to rule out a porcelain aorta. For longitudinal follow-up, stress echocardiography and cardiac magnetic resonance are both feasible modalities, as they are free of ionising radiation [20]. 


\subsection{Valvular heart disease}

RT-induced VHD is a common complication in cancers survivors irradiated with outdated RT-techniques, with incidences varying between 6-31\%[32-34]. In a large case-control study among 1,852 survivors of Hodgkin's disease irradiated between 1965-1995, the 30-year cumulative risk for any valvular involvement was $3.0 \%$ for a dose of $30 \mathrm{~Gy}$, which exponentially increased to $12.4 \%$ at doses of more than 40 Gy[35]. A splenectomy, at that time routinely performed in all patients with Hodgkin's disease for staging and treatment, more than doubled the risk for VHD (relative risk 2.3, 95\% CI 1.3-4.3), suggesting a synergetic role for inflammatory factors and RT in the pathophysiology of RT-induced VHD[35]. In a more recent study among lymphoma survivors treated with an autologous haematopoietic stem-cell transplant, chemotherapy and mediastinal RT between 1988 and 2008, mild valvular involvement was identified in almost $20 \%$ of the patients. Risk factors in this cohort for VHD were RT-dose>30 Gy, female gender, age >50 years at diagnosis and >3 lines of chemotherapy prior to stem-cell transplant[36].

Within one year after exposure, the first signs of RT-induced valvular damage can already be noted on echocardiography as valvular retractions and shortening of the mitral and aortic valve[37]. After ten to twenty years, the leaflets progressively become more fibrous leading to regurgitation and infrequently valvular stenosis. The combination of calcification of the aortic-mitral continuum and both the aortic and mitral valve, is the hallmark for RTinduced VHD on echocardiography[38]. Typically, the commissures and tips of the leaflets are relatively spared by RT (Figure 4), in contrast to rheumatic disease induced valvular dysfunction[39].

\subsection{Pericardial disease}

Prior to the 1970's, acute (pleuro)pericarditis was the most common CV complication after RT, which usually presented within four weeks after exposure[17]. Nowadays, with the implementation of more heart-sparing techniques, acute pericardial complications have become rare[13]. Nevertheless, chronic pericarditis is a relatively common side effect and may occur in $2.5 \%$ of cancer survivors after RT to the heart, typically manifesting as fibrous constrictive pericarditis[40].

The majority of the patients are diagnosed with asymptomatic pericardial effusion which may resolve spontaneously, although tamponade with hemodynamic compromise can occur[40]. An onset of pericardial effusion more than two years after RT-exposure is 
uncommon and requires exclusion of other etiologic factors, such as infection, CHF and carcinomatous pericarditis[17]. Additionally, the presence of post-RT pericardial complications is a sign of severe mediastinal exposure and therefore almost never an entity on its own[41]. Hence, screening for other comorbidities (i.e. CAD) is of crucial value.

\subsection{Conduction abnormalities and arrhythmias}

A large spectrum of conduction abnormalities and arrhythmias has been reported in irradiated cancer survivors, including sinus node dysfunction, atrioventricular block, and (supra)ventricular arrhythmias[42]. Of the intraventricular conduction disorders, right bundle branch block is more frequently observed due to its proximity to the radiation field[17]. Furthermore, T-wave inversions were reported in $45 \%$ of breast cancer patients six months after left-sided RT[43]. However, electrocardiograms normalised in the majority after ten years. A long-term follow-up of 44,423 early-stage breast cancer patients irradiated between 1982-2005 showed no significant increased risk for conduction abnormalities or severe ventricular arrhythmias in this cohort[44].

\section{Long-term systemic therapy-induced cardiovascular disease}

Exposure to systemic therapy (chemotherapy or targeted therapy) may pose a threat for CV health in cancer survivors in many ways. The most notorious long-term adverse effect is systemic therapy-related cardiac dysfunction. Table 1 provides an overview of current publications on long term cardiovascular complication in systemic therapy. We would like to emphasize that long-term follow-up data on CV complications are lacking in the majority of (modern) systemic therapy agents and that several of the correlations of CV complications after systemic therapy could be confounded by the concomitant use of antracyclines.

Other CV complications have been reported including arrhythmias, thromboembolic disease, and peripheral and cerebrovascular disease[13]. As these complications typically occur during or shortly after cardiotoxic chemotherapy, this falls outside the scope of this review.

\subsection{Left ventricular dysfunction and congestive heart failure}

Systemic therapy-related cardiac dysfunction is a relatively common complication and associated with clinical relevant CV morbidity and mortality, as well as reduced quality of life in cancer survivors[14,45]. The incidence and extent of left ventricular (LV) dysfunction is highly depended on the type of agent used, and in most cases the cumulative dose. 
Traditionally, two types of cardiotoxicity (type I and II) of systemic therapeutic agents were described, based on the potential reversibility of the induced myocardial dysfunction. Type I agents (e.g. anthracyclines) induce irreversible, dose-dependent myocardial dysfunction due to the induction of myocardial cell loss by apoptosis. Type II agents (e.g. trastuzumab) on the other hand have been found to be dose independent and typically these patients show a reversible LV dysfunction upon cessation of chemotherapy[14]. However, this classification is nowadays considered arbitrary, as new evidence among cancer survivors points out that patients treated with type II agents can develop persistent LV dysfunction[46].

Table 1. Systemic therapy agents associated with long-term LV dysfunction

\begin{tabular}{|l|l|}
\hline Chemotherapy agents & Daunorubicin[47] \\
\hline Anthracyclines & Doxorubicin[47] \\
\hline & Epirubicin[47] \\
\hline & Idarubicin [48] \\
\hline Alkylating agents & Mitoxantrone [48] \\
\hline Antimicrotubule agents & Cyclophosphamide* [49] \\
\hline & Docetaxel* [49] \\
\hline Targeted therapy agents & Paclitaxel* [50] \\
\hline Monoclonal antibodies & Trastuzumab[51] \\
\hline & Pertuzumab [52] \\
\hline & Bevacizumab** [53] \\
\hline Tyrosine kinase inhibitors & Sunitinib** [53] \\
\hline & Lapatinib [52] \\
\hline & Pazopanib**[53] \\
\hline & Sorafenib ** [53] \\
\hline & $* *$ potential long-term effects \\
\hline &
\end{tabular}

\section{Anthracycline-induced cardiotoxicity}

Since their introduction in the 1970's, anthracyclines constitute the cornerstone for the treatment of haematologic malignancies, breast cancer and many more[47]. In the first studies, incidence of anthracycline-induced cardiotoxicity (AIC) varied between 16- 
$23 \%[54,55]$. Several refinements in the following decades (e.g. reduction of cumulative dose and infusion scheme) aimed to reduce the incidence of clinically relevant cardiotoxicity. A large meta-analysis, pooling results from almost 50,000 cancer patients in the current era of adjusted chemotherapy protocols, reported an incidence in patients treated with anthracyclines for clinical overt cardiotoxicity and subclinical cardiotoxicity of 6\% (95\%CI 3\%-9\%) and $18 \%(95 \%$ CI $12 \%-24 \%)$ respectively[47].

The main underlying pathophysiological mechanism for AIC is presumably based on an interaction between anthracyclines and topoisomerase- $2 \beta$, which results in DNA damage in the cardiomyocytes followed by irreversible cell loss[56]. This risk of irreversible myocardial injury increases exponentially with cumulative anthracycline dose[57], which consequently led to restrictions of administered cumulative dose. Other additional risk factors associated with an increased risk for AIC are concomitant (or previous) thoracic RT, AfricanAmerican ancestry, age( $<15$ or $>65$ years), other (cardiac) comorbidities, female gender and the use of other cardiotoxic chemotherapeutic agents[47].

In the majority of patients receiving anthracyclines, AIC occurs within the first year after exposure[58]. However, clinical manifestation of AIC may appear years later, due to extensive compensatory mechanisms of the myocardium[59]. If AIC manifests as overt CHF, the response to treatment is poor and prognosis grim as the majority of patients die within two years $[60,61]$. On the other hand, if prompt heart failure treatment is initiated when the first sings of LV dysfunction are detected, LV function may show some reversibility[58].

\section{Non-anthracycline-induced cardiotoxicity}

Targeted therapy is also associated with the occurrence of treatment-related cardiac dysfunction. Trastuzumab, a monoclonal antibody against the human epidermal growth factor receptor-2 (HER-2), was introduced in the late 1990's as adjuvant therapy for women with HER-2 positive breast cancer[52]. The first study reported an alarming high incidence for severe LV dysfunction of $27 \%$ in women treated with a combination of anthracyclines, cyclophosphamide and trastuzumab[62]. Based on the hypothesis of synergetic cardiotoxicity, trastuzumab was from that point on administered separately from anthracycline treatment and it was advised to monitor left ventricular ejection fraction (LVEF) periodically[63]. A recent meta-analysis of almost 30,000 women treated with trastuzumab reported an incidence for severe cardiotoxicity of $3 \%$ (95\% CI 2.41-3.64) at three year follow-up[51]. Older age, a history of anthracycline administration, hypertension, obesity and diabetes are risk factors for trastuzumab-induced LV dysfunction[64,65]. 
In a recent randomised clinical trial (RCT), patients who received anthracyclines, cyclophosphamide and paclitaxel $(n=743)$ were compared with patients treated with the same chemotherapy regime, but with additional weekly trastuzumab (n=947). After seven years, cardiac events, defined as a LVEF decline of more than $10 \%$, cardiac death or manifestations of $\mathrm{CHF}$, occurred in $4 \%$ of the patients in the trastuzumab-arm versus $1.3 \%$ in the control $\operatorname{arm}[50]$. The risk for cardiac events greatly increased in patients who had a LVEF of 50-55\% after anthracyclines (HR 11.8, 95\%CI 3.9-36.0), suggesting that sequential trastuzumab administration might aggravate underlying myocardial damage. From all patients with LVEF decline, the majority recovered to baseline within twelve months. Nevertheless, a persistent decline in LVEF was reported in 3.8\% of the entire cohort, supporting the raising concern on the reversibility of trastuzumab-induced LV dysfunction on the long-term[50]. It is conceivable that trastuzumab might induce subclinical cardiotoxicity, which may go unrecognized by conventional LVEF measurements since novel cardiac imaging parameters are abnormal in an earlier stage[66]. The relevance of these observations, as well as the 'true' reversibility of these subclinical changes has to be explored in long-term follow-up studies.

Although long-term data on the cardiac outcome is lacking, it is worth mentioning the potential cardiotoxic effects of other newer targeted therapeutic agents. For pertuzumab and lapatinib, two more recent developed anti-HER2 agents, preliminary evidence suggest that cardiotoxicity might be similar or less than trastuzumab[52]. In a recent meta-analysis, antivascular endothelial growth factor (VEGF) agents, such as bevacizumab and sunitinib, are associated with increased cardiac dysfunction and cardiac ischaemia (OR 1.35 (95\%CI 1.061.70) and OR 2.83 (95\% CI 1.72-4.65)) compared to other systemic agents[53]. For CHF or fatal CV events, no significant risk was identified.

With respect to the long-term influence of adjuvant endocrine therapies on CVD in postmenopausal breast cancer survivors, the literature is conflicting. In a recent study aromatase inhibitors are associated with a higher risk of myocardial infarction compared with tamoxifen with a HR of 2.02 (95\% CI 1.16-3.53)[67]. A large observational study including over 13.000 patients with over 72.00 person-years, on the other hand, showed that the risk of the most serious cardiovascular events (cardiac ischaemia or stroke) was not elevated in aromatase inhibitor users compared with tamoxifen users[68]. Prospective follow-up studies will be needed to evaluate the precise long-term risk and the identification of potential baseline risk factors in these patients.

Androgen-deprivation therapy through surgical castration is equally effective as medical castration in controlling prostate cancer. This treatment, in particular long-term 
treatment, has been associated with a higher risk of peripheral artery disease as well as cardiac complications[69], especially in men with a cardiovascular medical history[70] while short term treatment does not show this association at long-term follow-up[71]. It should be noted that these data come from observational studies and are therefore prone to bias. A recent systematic review pointed out the lack of published, reliable evidence (in other words, no RCTs) describing the effects of androgen-deprivation therapy on CVD risk factors[72].

\section{$\underline{3.2 \text { Metabolic disorders }}$}

The awareness for late metabolic disorders among cancer survivors is increasing. Compared to age and gender matched controls, cancer survivors have higher incidences of hypertension (65.9\% vs $59.5 \%)$, diabetes mellitus (23.4\% vs $21.5 \%)$, dyslipidaemia (57.9\% vs $55.9 \%)$ and obesity (43.4\% vs $35.4 \%, \mathrm{p}<0.01$ for all)[9]. These disorders can be clustered by the term 'metabolic syndrome'. Although no consensus is reached on its exact criteria, the metabolic syndrome comprises risk factors of metabolic origin that often occur simultaneously[73]. Metabolic syndrome increases the risk for atherosclerotic disease and might therefore attribute to the overall $\mathrm{CV}$ risk in cancer survivors[74].

\section{Hypertension}

The most extensively studied component of the metabolic syndrome in cancer survivors is hypertension. Although multiple systemic therapeutic agents may induce hypertension, anti VEGF agents are most often associated with the onset of hypertension[75]. Depending on the type of anti VEGF agent and patients' characteristic, the incidence of hypertension induced by anti VEGF agents varies between 11-43\%[75]. In pooled results of 77 phase III and IV RCTs, VEGF-based chemotherapy is associated with a higher risk for hypertension and malignant hypertension, defined as >180/110 mm Hg with ORs of 5.28 (95\% CI 4.53-6.15) and 5.59 (95\% CI 4.67-6.69) respectively[53]. Although long-term outcomes of these patients are lacking, prompt antihypertensive therapy is recommended in order to minimize long-term complications[13].

Besides anti-VEGF agents, exposure to systemic treatment with cisplatin is associated with long-term hypertension, in particular in survivors of testicular cancer. In a twenty-year follow-up study of 990 survivors of testicular cancer, exposure to cisplatin-based chemotherapy or cisplatin-based chemotherapy combined with infradiaphragmatic RT significantly increased the use of antihypertensive drugs (OR 3.1 (95\%CI 1.9-5.2) and OR 3.7 (95\%CI 1.6-8.9) respectively)[76]. Treatment with RT alone did not increase the risk for 
hypertension. Furthermore, exposure to systemic cisplatin increased the prevalence of diabetes from $4.0 \%$ in the surgery group to $15.6 \%$. In addition, combination therapy with cisplatin and infradiaphragmatic RT also dramatically increased the risk for CAD (HR 5.3, 96\% CI 1.5-18.5) compared to patients treated with surgery alone suggesting a strong influence of metabolic disorders in the pathogenesis of CAD in these patients [76].

\section{Future perspectives}

In the past decades, significant improvements have been made in early cancer detection and treatment leading to improved prognosis in cancer patients. However, cancer treatment has detrimental long-term effects on $\mathrm{CV}$ health and early recognition of unwanted side effects is needed to initiate preventive treatment at an early stage in high-risk patients. Figure 5 provides a schematic overview of the available literature we set out in this overview article.

To answer the specific clinical problems cancer survivors nowadays face, prospective information is needed to fill the current gaps in knowledge. Of particular interest are the longterm outcomes of patients treated with non-anthracyclines as well as the role of conventional $\mathrm{CV}$ risk factors in cancer survivors. To do so, harmonisation of clinical healthcare pathways combined with a large registry of $\mathrm{CV}$ follow-up are necessary to make warranted clinical decisions. Promising initiatives so far include specialized cardio-oncology units [77] and long-term prospective screening programs for multiple $\mathrm{CV}$ - and other complications (e.g. kidney disease, depression), as is current practice in the Netherlands. It is anticipated that these and future multidisciplinary efforts will reduce the impact of anticancer treatment related side effects and thereby improve the long-term outcome of these patients considerably.

\section{Acknowledgements:}

Folkert W. Asselbergs is supported by a Dekker scholarship-Junior Staff Member 2014T001 - Netherlands Heart Foundation and UCL Hospitals NIHR Biomedical Research Centre.

All other authors have no disclosures

\section{References}

[1] K.D. Miller, R.L. Siegel, C.C. Lin, A.B. Mariotto, J.L. Kramer, J.H. Rowland, K.D. Stein, R. Alteri, A. Jemal, Cancer treatment and survivorship statistics, 2016., CA. Cancer J. Clin. 66 (2016) 271-289.

[2] J.S. de Moor, A.B. Mariotto, C. Parry, C.M. Alfano, L. Padgett, E.E. Kent, L. Forsythe, S. Scoppa, M. Hachey, J.H. Rowland, Cancer survivors in the United States: prevalence across the survivorship trajectory and implications for care., Cancer Epidemiol. Biomarkers Prev. 22 (2013) 561-570. 
[3] J. Ferlay, E. Steliarova-Foucher, J. Lortet-Tieulent, S. Rosso, J.W.W. Coebergh, H. Comber, D. Forman, F. Bray, Cancer incidence and mortality patterns in Europe: estimates for 40 countries in 2012., Eur. J. Cancer. 49 (2013) 1374-1403.

[4] B.M.P. Aleman, A.W. van den Belt-Dusebout, M.L. De Bruin, M.B. van 't Veer, M.H.A. Baaijens, J.P. de Boer, A.A.M. Hart, W.J. Klokman, M.A. Kuenen, G.M. Ouwens, H. Bartelink, F.E. van Leeuwen, Late cardiotoxicity after treatment for Hodgkin lymphoma., Blood. 109 (2007) 1878-1886.

[5] F.E. van Leeuwen, A.K. Ng, Long-term risk of second malignancy and cardiovascular disease after Hodgkin lymphoma treatment., Hematol. Am. Soc. Hematol. Educ. Progr. 2016 (2016) 323-330.

[6] C.K. Lee, D. Aeppli, M.E. Nierengarten, The need for long-term surveillance for patients treated with curative radiotherapy for Hodgkin's disease: University of Minnesota experience., Int. J. Radiat. Oncol. Biol. Phys. 48 (2000) 169-179.

[7] M.R. Keats, Y. Cui, S.A. Grandy, L. Parker, Cardiovascular disease and physical activity in adult cancer survivors: a nested, retrospective study from the Atlantic PATH cohort., J. Cancer Surviv. (2016).

[8] N.G. Zaorsky, T.M. Churilla, B.L. Egleston, S.G. Fisher, J.A. Ridge, E.M. Horwitz, J.M. Md, Causes of death among cancer patients., Ann. Oncol. Off. J. Eur. Soc. Med. Oncol. (2016).

[9] S.H. Armenian, L. Xu, B. Ky, C. Sun, L.T. Farol, S.K. Pal, P.S. Douglas, S. Bhatia, C. Chao, Cardiovascular Disease Among Survivors of Adult-Onset Cancer: A Community-Based Retrospective Cohort Study., J. Clin. Oncol. 34 (2016) 1122-1130.

[10] R.J. Koene, A.E. Prizment, A. Blaes, S.H. Konety, Shared Risk Factors in Cardiovascular Disease and Cancer., Circulation. 133 (2016) 1104-1114.

[11] H. Abdel-Qadir, P.C. Austin, D.S. Lee, E. Amir, J. V Tu, P. Thavendiranathan, K. Fung, G.M. Anderson, A Population-Based Study of Cardiovascular Mortality Following Early-Stage Breast Cancer., JAMA Cardiol. 2 (2017) 88-93.

[12] N. Pavo, M. Raderer, M. Hulsmann, et al. Cardiovascular biomarkers in patients with cancer and their association with all-cause mortality., Heart. 101 (2015) 1874-1880.

[13] J.L. Zamorano, P. Lancellotti, D. Rodriguez Munoz, et al. 2016 ESC Position Paper on cancer treatments and cardiovascular toxicity developed under the auspices of the ESC Committee for Practice Guidelines: The Task Force for cancer treatments and cardiovascular toxicity of the European Society of Cardiology (ESC, Eur. Heart J. 37 (2016) 2768-2801.

[14] M.S. Ewer, S.M. Ewer, Cardiotoxicity of anticancer treatments., Nat. Rev. Cardiol. 12 (2015) 547-558.

[15] M. Boerma, J. Wang, V. Sridharan, J.-M. Herbert, M. Hauer-Jensen, Pharmacological induction of transforming growth factor-beta1 in rat models enhances radiation injury in the intestine and the heart., PLoS One. 8 (2013) e70479.

[16] K.E. Cohn, J.R. Stewart, L.F. Fajardo, E.W. Hancock, Heart disease following radiation., Medicine (Baltimore). 46 (1967) 281-298.

[17] C. Jaworski, J.A. Mariani, G. Wheeler, D.M. Kaye, Cardiac complications of thoracic irradiation., J. Am. Coll. Cardiol. 61 (2013) 2319-2328.

[18] F.C. 3rd Brosius, B.F. Waller, W.C. Roberts, Radiation heart disease. Analysis of 16 young (aged 15 to 33 years) necropsy patients who received over 3,500 rads to the heart., Am. J. Med. 70 (1981) 519-530.

[19] F. Paris, Z. Fuks, A. Kang, P. Capodieci, G. Juan, D. Ehleiter, A. Haimovitz-Friedman, C. Cordon-Cardo, R. Kolesnick, Endothelial apoptosis as the primary lesion initiating intestinal radiation damage in mice., Science. 293 (2001) 293-297.

[20] P. Lancellotti, V.T. Nkomo, L.P. Badano, et al. Expert consensus for multi-modality 
imaging evaluation of cardiovascular complications of radiotherapy in adults: a report from the European Association of Cardiovascular Imaging and the American Society of Echocardiography., J. Am. Soc. Echocardiogr. 26 (2013) 1013-1032.

[21] R. Jurcut, J. Ector, K. Erven, H.F. Choi, J.-U. Voigt, Radiotherapy effects on systolic myocardial function detected by strain rate imaging in a left-breast cancer patient., Eur. Heart J. 28 (2007) 2966.

[22] J.C. Beukema, J.A. Langendijk, C.T. Muijs, Cardiac toxicity in the radiation treatment of esophageal cancer: an emerging concern., Future Cardiol. 11 (2015) 367-369.

[23] X. Ming, Y. Feng, C. Yang, W. Wang, P. Wang, J. Deng, Radiation-induced heart disease in lung cancer radiotherapy: A dosimetric update., Medicine (Baltimore). 95 (2016)

[24] M.T. Nolan, D.J. Russell, K. Negishi, T.H. Marwick, Meta-Analysis of Association Between Mediastinal Radiotherapy and Long-Term Heart Failure., Am. J. Cardiol. 118 (2016) 1685-1691.

[25] F.A. van Nimwegen, M. Schaapveld, C.P.M. Janus, A.D.G. Krol, E.J. Petersen, J.M.M. Raemaekers, W.E.M. Kok, B.M.P. Aleman, F.E. van Leeuwen, Cardiovascular disease after Hodgkin lymphoma treatment: 40-year disease risk., JAMA Intern. Med. 175 (2015) 1007-1017.

[26] S.L. Hancock, M.A. Tucker, R.T. Hoppe, Factors affecting late mortality from heart disease after treatment of Hodgkin's disease., JAMA. 270 (1993) 1949-1955.

[27] L.S. Constine, R.G. Schwartz, D.E. Savage, V. King, A. Muhs, Cardiac function, perfusion, and morbidity in irradiated long-term survivors of Hodgkin's disease., Int. J. Radiat. Oncol. Biol. Phys. 39 (1997) 897-906.

[28] S.C. Darby, M. Ewertz, P. McGale, A.M. Bennet, U. Blom-Goldman, D. Bronnum, C. Correa, D. Cutter, G. Gagliardi, B. Gigante, M.-B. Jensen, A. Nisbet, R. Peto, K. Rahimi, C. Taylor, P. Hall, Risk of ischemic heart disease in women after radiotherapy for breast cancer., N. Engl. J. Med. 368 (2013) 987-998.

[29] P.T. McEniery, K. Dorosti, W.A. Schiavone, T.J. Pedrick, W.C. Sheldon, Clinical and angiographic features of coronary artery disease after chest irradiation., Am. J. Cardiol. 60 (1987) 1020-1024.

[30] G. Nilsson, L. Holmberg, H. Garmo, O. Duvernoy, I. Sjogren, B. Lagerqvist, C. Blomqvist, Distribution of coronary artery stenosis after radiation for breast cancer., J. Clin. Oncol. 30 (2012) 380-386.

[31] E.M. van Leeuwen-Segarceanu, W.-J.W. Bos, L.D.A. Dorresteijn, B.J.W.M. Rensing, J.A.S. van der Heyden, O.J.M. Vogels, D.H. Biesma, Screening Hodgkin lymphoma survivors for radiotherapy induced cardiovascular disease., Cancer Treat. Rev. 37 (2011) 391-403.

[32] T. Wethal, M.-B. Lund, T. Edvardsen, S.D. Fossa, A.H. Pripp, H. Holte, J. Kjekshus, A. Fossa, Valvular dysfunction and left ventricular changes in Hodgkin's lymphoma survivors. A longitudinal study., Br. J. Cancer. 101 (2009) 575-581.

[33] M.B. Lund, H. Ihlen, B.M. Voss, A.F. Abrahamsen, O. Nome, J. Kongerud, M. Stugaard, K. Forfang, Increased risk of heart valve regurgitation after mediastinal radiation for Hodgkin's disease: an echocardiographic study., Heart. 75 (1996) 591595.

[34] M.C. Hull, C.G. Morris, C.J. Pepine, N.P. Mendenhall, Valvular dysfunction and carotid, subclavian, and coronary artery disease in survivors of hodgkin lymphoma treated with radiation therapy., JAMA. 290 (2003) 2831-2837.

[35] D.J. Cutter, M. Schaapveld, S.C. Darby, M. Hauptmann, F.A. van Nimwegen, A.D.G. Krol, C.P.M. Janus, F.E. van Leeuwen, B.M.P. Aleman, Risk of valvular heart disease after treatment for Hodgkin lymphoma., J. Natl. Cancer Inst. 107 (2015). 
[36] K. Murbraech, T. Wethal, K.B. Smeland, H. Holte, J.H. Loge, E. Holte, A. Rosner, H. Dalen, C.E. Kiserud, S. Aakhus, Valvular Dysfunction in Lymphoma Survivors Treated With Autologous Stem Cell Transplantation: A National Cross-Sectional Study., JACC. Cardiovasc. Imaging. 9 (2016) 230-239.

[37] J.R. Cuomo, G.K. Sharma, P.D. Conger, N.L. Weintraub, Novel concepts in radiationinduced cardiovascular disease., World J. Cardiol. 8 (2016) 504-519.

[38] D. Hering, L. Faber, D. Horstkotte, Echocardiographic features of radiation-associated valvular disease., Am. J. Cardiol. 92 (2003) 226-230.

[39] L. Krapf, J. Dreyfus, C. Cueff, L. Lepage, E. Brochet, A. Vahanian, D. MessikaZeitoun, Anatomical features of rheumatic and non-rheumatic mitral stenosis: potential additional value of three-dimensional echocardiography., Arch. Cardiovasc. Dis. 106 (2013) 111-115.

[40] M.M. Applefeld, J.F. Cole, S.H. Pollock, F.J. Sutton, R.G. Slawson, R.T. Singleton, P.H. Wiernik, The late appearance of chronic pericardial disease in patients treated by radiotherapy for Hodgkin's disease., Ann. Intern. Med. 94 (1981) 338-341.

[41] J.D. Groarke, P.L. Nguyen, A. Nohria, R. Ferrari, S. Cheng, J. Moslehi, Cardiovascular complications of radiation therapy for thoracic malignancies: the role for non-invasive imaging for detection of cardiovascular disease., Eur. Heart J. 35 (2014) 612-623.

[42] R.L. Larsen, R.I. Jakacki, V.L. Vetter, A.T. Meadows, J.H. Silber, G. Barber, Electrocardiographic changes and arrhythmias after cancer therapy in children and young adults., Am. J. Cardiol. 70 (1992) 73-77.

[43] L.E. Strender, J. Lindahl, L.E. Larsson, Incidence of heart disease and functional significance of changes in the electrocardiogram 10 years after radiotherapy for breast cancer., Cancer. 57 (1986) 929-934.

[44] J.C. Rehammar, J.B. Johansen, M.-B. Jensen, L. Videbaek, O.D. Jorgensen, E. Lorenzen, M. Ewertz, Risk of pacemaker or implantable cardioverter defibrillator after radiotherapy for early-stage breast cancer in Denmark, 1982-2005., Radiother. Oncol. 122 (2017) 60-65.

[45] C.L. Cox, S.N. Rai, D. Rosenthal, S. Phipps, M.M. Hudson, Subclinical late cardiac toxicity in childhood cancer survivors: impact on self-reported health., Cancer. 112 (2008) 1835-1844.

[46] M.L. Telli, S.A. Hunt, R.W. Carlson, A.E. Guardino, Trastuzumab-related cardiotoxicity: calling into question the concept of reversibility., J. Clin. Oncol. 25 (2007) 3525-3533.

[47] M. Lotrionte, G. Biondi-Zoccai, A. Abbate, G. Lanzetta, F. D’Ascenzo, V. Malavasi, M. Peruzzi, G. Frati, G. Palazzoni, Review and meta-analysis of incidence and clinical predictors of anthracycline cardiotoxicity., Am. J. Cardiol. 112 (2013) 1980-1984.

[48] P. Pellicori, A. Calicchia, F. Lococo, G. Cimino, C. Torromeo, Subclinical anthracycline cardiotoxicity in patients with acute promyelocytic leukemia in longterm remission after the AIDA protocol., Congest. Heart Fail. 18 (2012) 217-221.

[49] J.R. Mackey, M. Martin, T. Pienkowski, et al. Adjuvant docetaxel, doxorubicin, and cyclophosphamide in node-positive breast cancer: 10-year follow-up of the phase 3 randomised BCIRG 001 trial., Lancet. Oncol. 14 (2013) 72-80.

[50] E.H. Romond, J.-H. Jeong, P. Rastogi, et al. Seven-year follow-up assessment of cardiac function in NSABP B-31, a randomized trial comparing doxorubicin and cyclophosphamide followed by paclitaxel (ACP) with ACP plus trastuzumab as adjuvant therapy for patients with node-positive, human epidermal gr, J. Clin. Oncol. 30 (2012) 3792-3799.

[51] S. Mantarro, M. Rossi, M. Bonifazi, R. D’Amico, C. Blandizzi, C. La Vecchia, E. Negri, L. Moja, Risk of severe cardiotoxicity following treatment with trastuzumab: a 
meta-analysis of randomized and cohort studies of 29,000 women with breast cancer., Intern. Emerg. Med. 11 (2016) 123-140.

[52] N.F. Ponde, M. Lambertini, E. de Azambuja, Twenty years of anti-HER2 therapyassociated cardiotoxicity., ESMO Open. 1 (2016) e000073. d

[53] H. Abdel-Qadir, J.-L. Ethier, D.S. Lee, P. Thavendiranathan, E. Amir, Cardiovascular toxicity of angiogenesis inhibitors in treatment of malignancy: A systematic review and meta-analysis., Cancer Treat. Rev. 53 (2017) 120-127.

[54] A.C. Gilladoga, C. Manuel, C.T. Tan, N. Wollner, S.S. Sternberg, M.L. Murphy, The cardiotoxicity of adriamycin and daunomycin in children., Cancer. 37 (1976) 10701078.

[55] L.J. Steinherz, P.G. Steinherz, C.T. Tan, G. Heller, M.L. Murphy, Cardiac toxicity 4 to 20 years after completing anthracycline therapy., JAMA. 266 (1991) 1672-1677.

[56] P. Vejpongsa, E.T.H. Yeh, Prevention of anthracycline-induced cardiotoxicity: Challenges and opportunities, J. Am. Coll. Cardiol. 64 (2014) 938-945.

[57] S.M. Swain, F.S. Whaley, M.S. Ewer, Congestive heart failure in patients treated with doxorubicin: a retrospective analysis of three trials., Cancer. 97 (2003) 2869-2879.

[58] D. Cardinale, A. Colombo, G. Bacchiani, I. Tedeschi, C.A. Meroni, F. Veglia, M. Civelli, G. Lamantia, N. Colombo, G. Curigliano, C. Fiorentini, C.M. Cipolla, Early detection of anthracycline cardiotoxicity and improvement with heart failure therapy., Circulation. 131 (2015) 1981-1988.

[59] J.C. Plana, M. Galderisi, A. Barac, et al. Expert consensus for multimodality imaging evaluation of adult patients during and after cancer therapy: a report from the American Society of Echocardiography and the European Association of Cardiovascular Imaging., Eur. Heart J. Cardiovasc. Imaging. 15 (2014) 1063-1093.

[60] D. Cardinale, A. Colombo, G. Lamantia, N. Colombo, M. Civelli, G. De Giacomi, M. Rubino, F. Veglia, C. Fiorentini, C.M. Cipolla, Anthracycline-induced cardiomyopathy: clinical relevance and response to pharmacologic therapy., J. Am. Coll. Cardiol. 55 (2010) 213-220. .

[61] G.M. Felker, R.E. Thompson, J.M. Hare, R.H. Hruban, D.E. Clemetson, D.L. Howard, K.L. Baughman, E.K. Kasper, Underlying causes and long-term survival in patients with initially unexplained cardiomyopathy., N. Engl. J. Med. 342 (2000) 1077-1084. .

[62] D.J. Slamon, B. Leyland-Jones, S. Shak, H. Fuchs, V. Paton, A. Bajamonde, T. Fleming, W. Eiermann, J. Wolter, M. Pegram, J. Baselga, L. Norton, Use of chemotherapy plus a monoclonal antibody against HER 2 for metastatic breast cancer that overexpresses HER2., N. Engl. J. Med. 344 (2001) 783-792. ..

[63] J.J. Moslehi, Cardiovascular Toxic Effects of Targeted Cancer Therapies., N. Engl. J. Med. 375 (2016) 1457-1467. .

[64] Z. Jawa, R.M. Perez, L. Garlie, M. Singh, R. Qamar, B.K. Khandheria, A. Jahangir, Y. Shi, Risk factors of trastuzumab-induced cardiotoxicity in breast cancer: A metaanalysis., Medicine (Baltimore). 95 (2016) e5195. .

[65] C. Guenancia, A. Lefebvre, D. Cardinale, A.F. Yu, S. Ladoire, F. Ghiringhelli, M. Zeller, L. Rochette, Y. Cottin, C. Vergely, Obesity As a Risk Factor for Anthracyclines and Trastuzumab Cardiotoxicity in Breast Cancer: A Systematic Review and MetaAnalysis., J. Clin. Oncol. 34 (2016) 3157-3165. .

[66] N. Fallah-Rad, J.R. Walker, A. Wassef, M. Lytwyn, S. Bohonis, T. Fang, G. Tian, I.D.C. Kirkpatrick, P.K. Singal, M. Krahn, D. Grenier, D.S. Jassal, The utility of cardiac biomarkers, tissue velocity and strain imaging, and cardiac magnetic resonance imaging in predicting early left ventricular dysfunction in patients with human epidermal growth factor receptor II-positive breast cancer treated with a, J. Am. Coll. Cardiol. 57 (2011) 2263-2270. . 
[67] H. Abdel-Qadir, E. Amir, H.D. Fischer, L. Fu, P.C. Austin, P.J. Harvey, P.A. Rochon, D.S. Lee, G.M. Anderson, The risk of myocardial infarction with aromatase inhibitors relative to tamoxifen in post-menopausal women with early stage breast cancer., Eur. J. Cancer. 68 (2016) 11-21.

[68] R. Haque, J. Shi, J.E. Schottinger, J. Chung, C. Avila, B. Amundsen, X. Xu, A. Barac, R.T. Chlebowski, Cardiovascular Disease After Aromatase Inhibitor Use., JAMA Oncol. 2 (2016) 1590-1597.

[69] M. Sun, T.K. Choueiri, O.-P.R. Hamnvik, M.A. Preston, G. De Velasco, W. Jiang, S. Loeb, P.L. Nguyen, Q.-D. Trinh, Comparison of Gonadotropin-Releasing Hormone Agonists and Orchiectomy: Effects of Androgen-Deprivation Therapy., JAMA Oncol. 2 (2016) 500-507.

[70] S. O'Farrell, H. Garmo, L. Holmberg, J. Adolfsson, P. Stattin, M. Van Hemelrijck, Risk and timing of cardiovascular disease after androgen-deprivation therapy in men with prostate cancer., J. Clin. Oncol. 33 (2015) 1243-1251.

[71] J.C. Voog, R. Paulus, W.U. Shipley, M.R. Smith, D.G. McGowan, C.U. Jones, J.-P. Bahary, K.L. Zeitzer, L. Souhami, M.H. Leibenhaut, M. Rotman, S.M. Husain, E. Gore, A. Raben, S. Chafe, H.M. Sandler, J.A. Efstathiou, Cardiovascular Mortality Following Short-term Androgen Deprivation in Clinically Localized Prostate Cancer: An Analysis of RTOG 94-08., Eur. Urol. 69 (2016) 204-210.

[72] M.L. Romo, A.M. McCrillis, J. Brite, D. Reales, J.B. Dowd, C.M. Schooling, Pharmacologic androgen deprivation and cardiovascular disease risk factors: a systematic review., Eur. J. Clin. Invest. 45 (2015) 475-484.

[73] K.G.M.M. Alberti, P. Zimmet, J. Shaw, Metabolic syndrome--a new world-wide definition. A Consensus Statement from the International Diabetes Federation., Diabet. Med. 23 (2006) 469-480. .

[74] E.C. de Haas, S.F. Oosting, J.D. Lefrandt, B.H. Wolffenbuttel, D.T. Sleijfer, J.A. Gietema, The metabolic syndrome in cancer survivors., Lancet. Oncol. 11 (2010) 193203. .

[75] A. Milan, E. Puglisi, L. Ferrari, G. Bruno, I. Losano, F. Veglio, Arterial hypertension and cancer., Int. J. Cancer. 134 (2014) 2269-2277..

[76] H.S. Haugnes, T. Wethal, N. Aass, O. Dahl, O. Klepp, C.W. Langberg, T. Wilsgaard, R.M. Bremnes, S.D. Fossa, Cardiovascular risk factors and morbidity in long-term survivors of testicular cancer: a 20-year follow-up study., J. Clin. Oncol. 28 (2010) 4649-4657. .

[77] S. Barros-Gomes, J Herrmann, S.L. Mulvagh, A. Lerman, G. Lin, H.R. Villarraga. Rationale for setting up a cardio-oncology unit: our experience at Mayo Clinic. CardioOncology 2016 2:5 